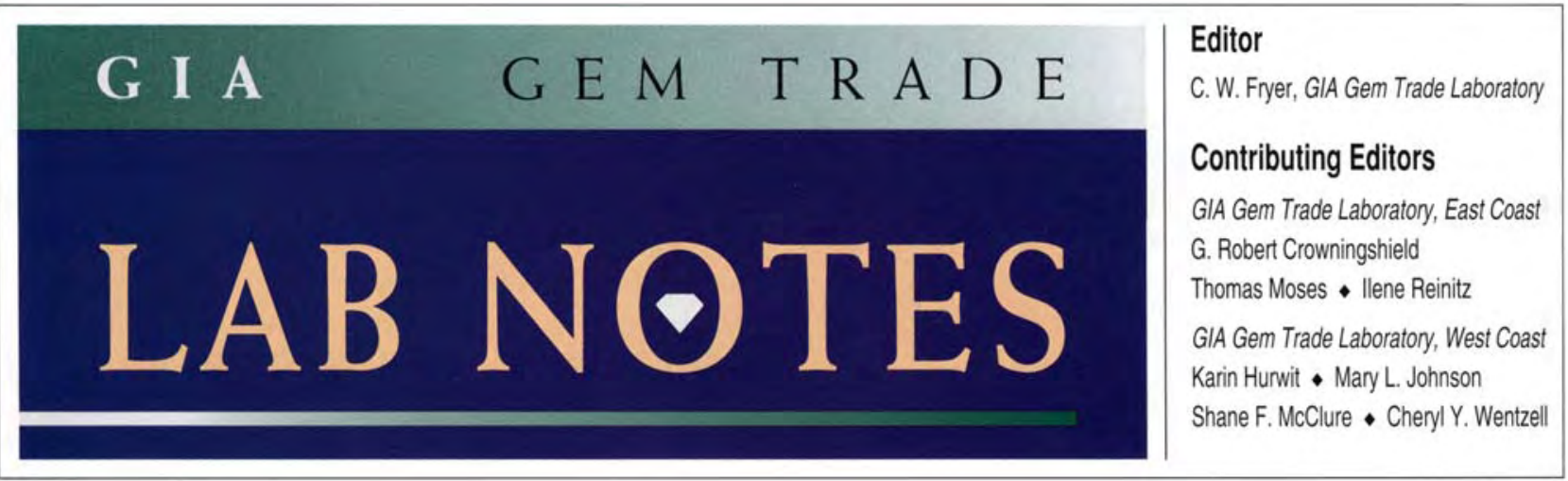

\section{Etched CHALCEDONY}

In early 1997, a 90.5-mm-long mottled brownish and yellowish orange pierced carving was submitted to the West Coast laboratory for identification. Because the surface was covered with an intricate pattern of swirls, we could not obtain an R.I. reading. Also, the large size precluded specific gravity testing by the hydrostatic method. The carving did have an aggregate

Figure 1. Details of the surface structure of a chalcedony carving show that the material has been selectively etched, probably by a hydrofluoric acid solution. Note the agate-like bands crossing the etched channels in the carving. Magnified $10 \times$.

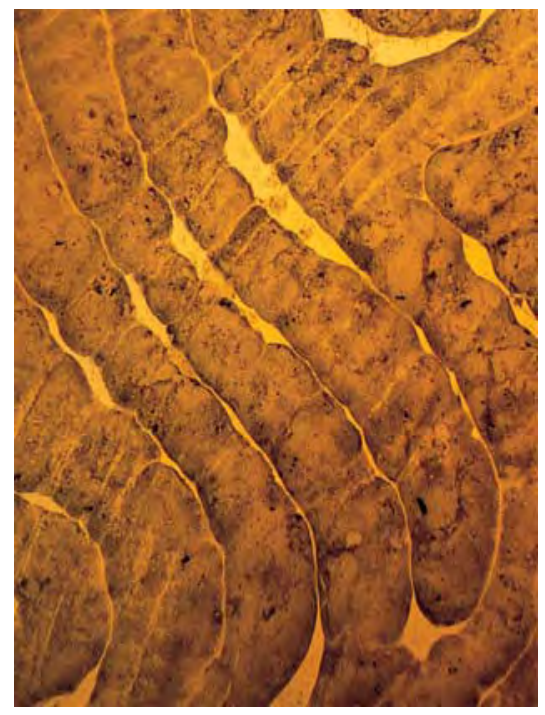

optic character, revealed no spectrum when viewed with a handheld spectroscope, and was inert to both longand short-wave ultraviolet radiation. However, an X-ray powder diffraction pattern (produced from a minute amount of material taken from an inconspicuous spot on the carving) matched that of quartz.

In addition, with magnification and diffused lighting conditions, we saw a raised agate-like banding in many areas of the carving. We also noted that areas within the carved channels appeared to have been dissolved, with most of the ridges between the channels reduced to sharp edges (figure 1). This appearance indicated that the carving had been etched with a caustic solution, such as hydrofluoric acid (which is extremely dangerous). This would also explain the raised nature of the agate-like banding, since acid etching is known to bring out structural irregularities in aggregates. On the basis of this structure and the diffraction pattern, we identified the material as chalcedony. We suspect that this carving was etched to give it an antique appearance.

MLJ, SFM, Dino DeGhionno, and Philip Owens

\section{DIAMOND}

\section{With “Additional" Color}

Occasionally we encounter diamonds with overall characteristic color appearances that result from factors

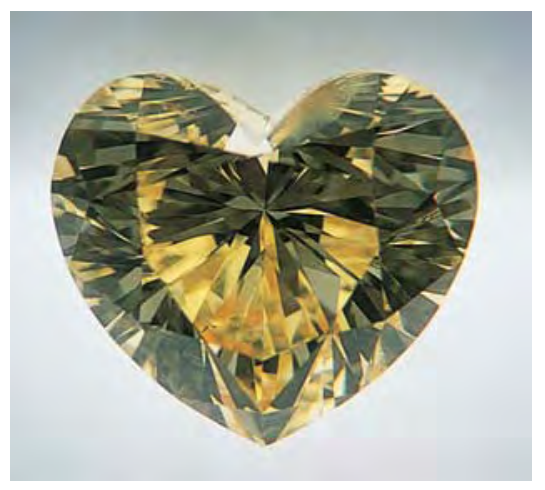

Figure 2. A distinctive orangecolored area can be seen under the table and toward the tip of this $1.20 \mathrm{ct}$ heart-shaped Fancy yellow diamond.

other than those that color the diamond itself. For example, inclusions are often responsible for the apparent body color (see, e.g., Fall 1995 Lab Notes, pp. 197-198, and R. C. Kammerling et al., "An Investigation of a Suite of Black Diamond Jewelry," Winter 1990 Gems Æ) Gemology, pp. 282-287). In some cases, however such factors do not affect the overall color, but they do produce an "additional" color. This is well illustrated by a 1.20 ct heart shape recently submitted to the East Coast lab.

Editor's note: The initials at the end of each item identify the contributing editor(s) who provided that item.

Gems \& Gemology, Vol. 33, No. 2, pp. 134-141

(C) 1997 Gemological Institute of America 


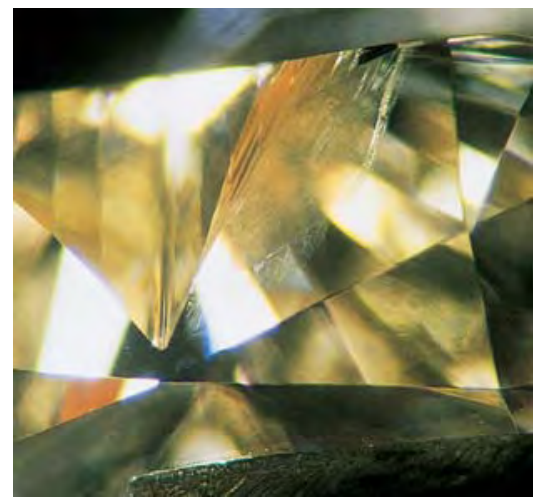

Figure 3. Stains in fractures, such as this orange limonitic stain in the stone shown in figure 2, can impart an additional aspect to the color appearance of a diamond. Magnified 32×.

During color grading, we observed a distinct patch of orange that was separate from the yellow body color (figure 2). Closer examination revealed an orange limonitic stain that was confined to a fracture (figure 3). When the stone was viewed in the standard face-up color-grading position, the orange reflections from this stain were seen under the table edge toward the tip of the heart.
In situations such as this where the additional color component does not affect the color grade, a comment is included in the report. We graded this stone Fancy yellow and added the comment: "The orangy color appearance of this diamond is influenced by a stain in a fracture." In our experience, boiling in acid will usually remove such stains. John King

\section{Rough with \\ Contrasting Colors}

In recent years, we have been encouraging diamond manufacturers to show us diamonds with a strong green color component when they are still rough or when blocked, because examination of the original crystal surface of such diamonds can be very helpful in determining whether the green component is natural or caused by laboratory treatment. We document the diamond's properties and use these observations along with those of the finished stone when it is submitted for a laboratory grading report. In keeping with this practice, one manufacturer brought us the 2.14 ct piece of green-and-pink rough shown in figure 4.

The slightly distorted octahedron had the properties of a natural-color pink diamond, especially the pink graining, an absorption at $415 \mathrm{~nm}$ visible with a desk-model spectroscope, and blue fluorescence to long-wave UV. The green came from strongly colored green radiation stains that lined several fractures. With magnification, one of these stained fractures showed the spotted appearance typical of natural radiation stains (figure 5). Because these stains were on the surfaces of the fractures and did not penetrate the body of the stone, we predicted that it would be difficult to retain any green color after cutting and polishing - unless one or more of the color-causing fractures were also retained. Ultimately, the rough was cut into a $1.07 \mathrm{ct}$ heart shape that was color graded Fancy Intense purplepink. All of the fractures were removed during cutting and, as we had predicted, there was no green color in the finished stone.

We have reported radiation stains on colored diamonds before (e.g., in Lab Notes: Summer 1991, p. 110; Fall 1991, pp. 174-175; Winter 1991, p. 249; and Winter 1993, p. 279|, but we had not previously seen a stone with such contrasting colors. This example
Figure 4. This $2.14 \mathrm{ct}$ distorted octahedral diamond crystal shows strongly saturated areas of both pink and green.

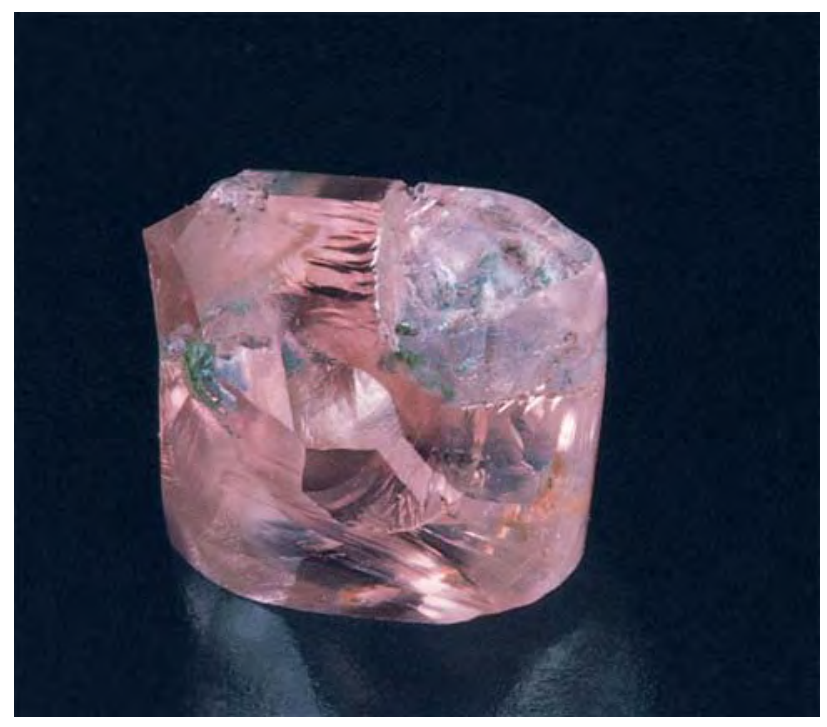

Figure 5. With $30 \times$ magnification, we saw that fractures in the diamond shown in figure 4 have the streaked and spotted appearance typical of natural radiation stains. The green color is largely confined to fracture surfaces.

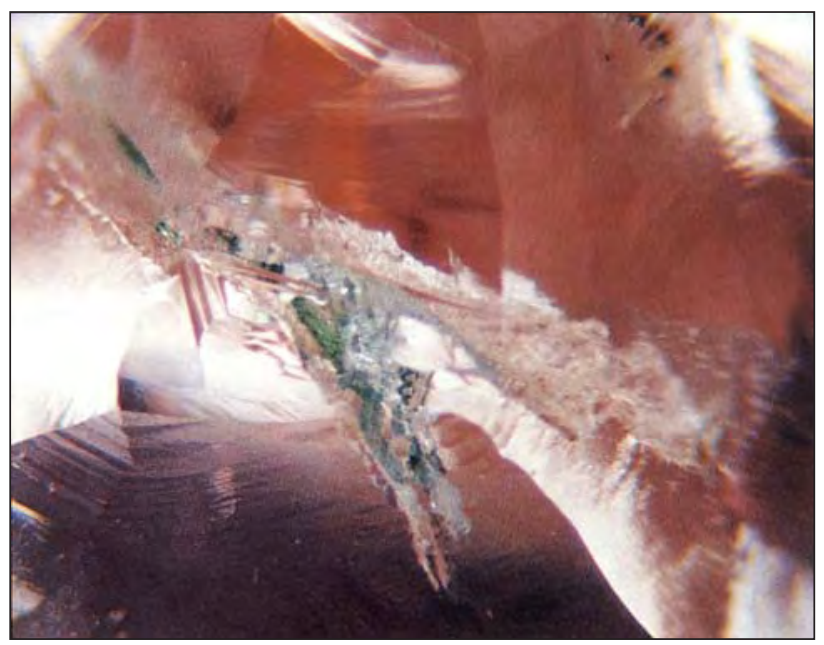




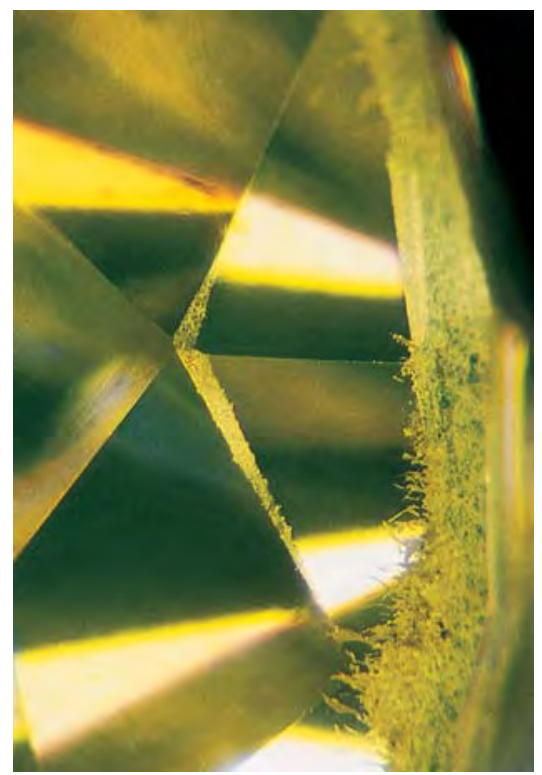

Figure 6. The facet junctions on either side of the upper girdle facets of this $0.59 \mathrm{ct}$ treated green-yellow round brilliant diamond have a frosted, burned appearance that is indicative of heat treatment. Magnified 63×.

is a reminder that any diamondregardless of its color or inherent properties-may be exposed to natural radiation and thus develop green stains.

$I R$

\section{Treated-Color Yellow Diamonds With Green Graining}

Last fall, the East Coast laboratory saw a number of unusual treatedcolor diamonds. All round brilliants under $1 \mathrm{ct}$, they displayed highly saturated greenish yellow to yellow-green hues. In the past, diamonds with these colors have been relatively rare, so we were surprised to see several at one time. There were two components to the color in these stones: a saturated yellow body color and a strong green luminescence to visible light. The latter is sometimes referred to as "green transmission." Both the color and the luminescence showed zoned distribution, with brownish yellow graining visible against a pervasive paler yellow body color; only the graining exhibited green luminescence to a strong visible light source. Prior to examining these particular diamonds, we had believed that green transmission luminescence confined to colored graining was only associated with diamonds of natural color (see, e.g., R. Crowningshield, "Developments and Highlights at GIA's Lab in New York," Spring 1979 Gems «) Gemology, pp. 153-154). However, other features of these stones proved that this is not always the case.

With magnification, we observed several features that were similar to those observed in diamonds we know to have been burned (see, e.g., Spring 1992 Lab Notes, p. 53). Several of the diamonds showed pitted, frosted surfaces at facet junctions on the crown (figure 6). All of the stones had heavily bearded girdles, and the short fractures in the bearding also had a frosted appearance. Larger fractures, seen in a few of the diamonds, had a similar appearance along their outer edges. At first glance, all the girdles appeared to have been bruted. In reflected light, however, many of them showed remnants of girdle facets, indicating instead that they had been burned. These observations prompted us to look more closely at the other properties relevant to determining the origin of color.

The spectra observed with a desk-model spectroscope showed absorption lines at 415 and $503 \mathrm{~nm}$, with a dark region between 465 and $494 \mathrm{~nm}$, and pairs of absorption and bright emission lines at 513 and 518 $\mathrm{nm}$. (A good example of such a spectrum in a natural-color greenish yellow diamond appears in Gems 4 Gemology, Spring 1961, p. 152.) The fluorescence to long-wave UV radiation was a mixture of greenish yellow and blue, a common response for diamonds with strong green transmission; unlike known natural-color diamonds of this type that we have examined, though, the fluorescence of these stones was strongly chalky.

The spectrophotometer showed us that, in addition to the lines seen with the spectroscope, most of these diamonds also had a weak peak at 637 $\mathrm{nm}$. We did not see features that we would expect in treated-color yellow or green diamonds, such as the $595 \mathrm{~nm}$ line or the GR1 line (741 nm). (The H1b and H1c peaks, which, when present, are seen with mid-infrared FTIR spectroscopy, were also not seen in these stones.) Most significantly, all the diamonds had a peak at $985 \mathrm{~nm}$ (in the near-infrared, and thus not visible with a spectroscope), which was weak in some stones and strong in others. This peak at $985 \mathrm{~nm}$ is caused by the H2 center (see S. C. Lawton et al., 'The 'H2' Optical Transition in Diamond: The Effects of Uniaxial Stress Perturbations, Temperature, and Isotopic Substitution," Journal of Physics: Condensed Matter, Vol. 4, 1992, pp. 3439-3452). It has never been observed in a natural-color diamond. However, the $\mathrm{H} 2$ peak has been produced by irradiation and heat treatment to extremely high temperatures (greater than $1400^{\circ} \mathrm{C}$ ). Such heat treatment would account for the "burned" surface features we observed in these stones with the microscope.

In general, it is not possible to heat a diamond to these high temperatures for more than a few minutes without a stabilizing factor such as an inert atmosphere or high confining pressure. This suite of diamonds may indicate that a "new" diamond treatment is being used in the trade. Although some factors (such as evidence of burning) may suggest that yellow to yellow-green diamonds have been treated in this fashion, the $985 \mathrm{~nm}$ peak is the strongest proof that such treatment has taken place.

IR and TM

\section{EMERALD, \\ An Unusual Assembled Imitation}

One of the most elaborate and natural-appearing assembled stones to come to our attention was submitted to the East Coast lab for identification and determination of whether the "emerald" was treated. Weighing just under $1 \mathrm{ct}$, the stone appeared to be an attractive, moderately included 


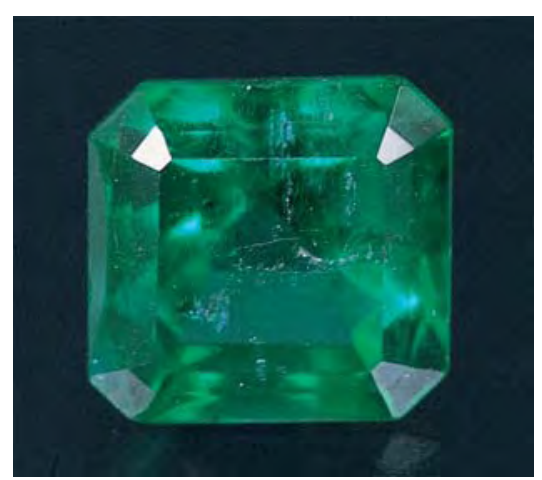

Figure 7. This $5.95 \times 5.92 \times 5.23$ $\mathrm{mm}$ assemblage imitated a fine emerald of $0.98 \mathrm{ct}$.

emerald (figure 7). The refractive index reading on the table facet was 1.578-1.583, the expected value for natural emerald. A quick microscopic examination through the table revealed the jardin typical of natural emerald, as well as a few orange areas in fractures; none of these inclusions extended deep into the pavilion of the stone. As part of the procedure to check for treatment, we exposed the stone to long-wave ultraviolet radiation in a darkened room. (Emerald may fluoresce red or remain inert, whereas some of the materials used to fill fractures in emerald fluoresce yellow.) The fluorescence reaction was definitely not that of emerald, either filled or unfilled: The sample glowed a strong chalky yellow-green! With magnification and UV radiation, we could see that the fluorescence was confined to the pavilion surface.

Because there were no chrome lines in the absorption spectrum, we searched for additional clues to the cause of the strong green color. Immersion of the piece in water revealed a most unusual combination. As seen in figure 8, within the original stone there is the ghost-like outline of another faceted stone, the pavilion of which is considerably shallower than the assemblage as a whole. The dark green color gradually diminishes from the culet upward, ending just below the girdle. Because the refractive index of the pavilion

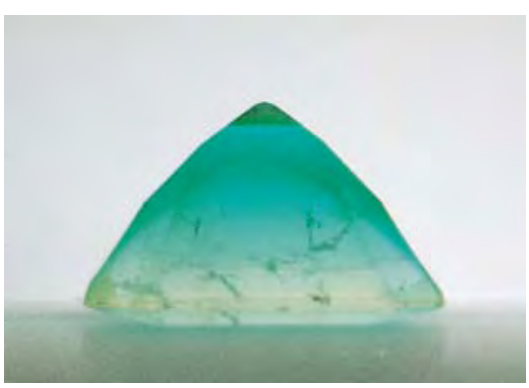

Figure 8. Immersed in water, the assembled nature of the stone shown in figure 7 is evident: a faceted gem with a shallow pavilion that has been overlaid with a green material.

was 1.50 and the hardness was low on the Mohs scale (as noted with magnification when the needle probe indented a minute area on the surface), we concluded that this portion of the assemblage was a plastic. We were unable to determine if there was even a faint green color in the natural beryl portion of the stone. After careful observation, we determined that the assemblage consisted of a fully faceted natural beryl with a shallow pavilion over which a green plastic had been added to impart the color and add apparent depth. Certainly, if such a stone were mounted, a cursory examination might result in a devastating misidentification.

GRC

\section{Star FELDSPAR}

Asteriated feldspars were once common in the gem trade, but we had not seen one in the GIA Gem Trade Laboratory for several years. In spring 1997, a 64.48 ct dark brown oval double cabochon was submitted to the West Coast lab for identification. It measured about $28.92 \times 19.20 \times 16.22$ $\mathrm{mm}$. In addition to a four-rayed star, it exhibited two parallel bands of chatoyancy (one of which is too faint to be seen in figure 9). The stone had a (spot) refractive index of 1.52 and a specific gravity (measured hydrostatically) of 2.60. It was inert to longwave UV and fluoresced very weak red to short-wave UV. All these prop- erties were consistent with a feldspar. An EDXRF spectrum, taken to understand the stone better, detected major amounts of $\mathrm{Al}, \mathrm{Si}$, and $\mathrm{K}$, and lesser amounts of $\mathrm{Na}, \mathrm{Fe}, \mathrm{Ga}, \mathrm{Sr}, \mathrm{Ba}, \mathrm{Rb}$, and $\mathrm{Pb}$. Thus, we determined that it was a potassium feldspar, but we could not establish which feldspar-orthoclase, microcline, or sanidine-without further testing, which was not authorized by the client.

With magnification, we saw platy inclusions-possibly micathroughout the stone. These appeared to be partially oriented along various planes in the cabochon, one being parallel to the girdle. However, the inclusions were too large and too poorly oriented to account for either of the optical effects. We do not recall seeing another fashioned feldspar gemstone that showed asterism associated with chatoyancy.

MLJ, SFM, Dino DeGhionno, and Philip Owens

\section{JADEITE JADE}

\section{Beads of an Unusual Color}

An attractive necklace of predominantly brown-and-white (with lesser amounts of green) variegated beads (figure 10), which a client purchased recently in Tucson, was submitted to the East Coast lab for identification. We identified the beads as jadeite on the basis of standard gemological test-

Figure 9. This 64.48 ct feldspar cabochon shows a four-rayed star and two chatoyant bands, one of which is much fainter than the other.

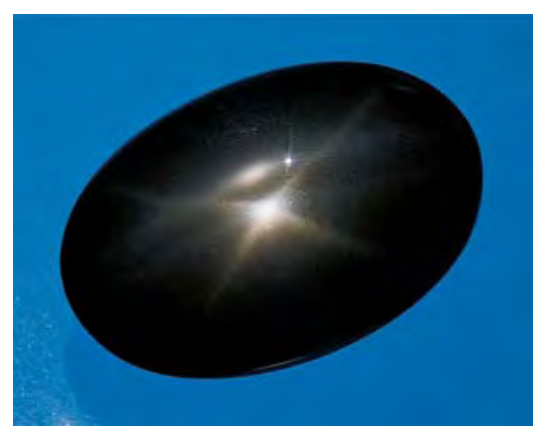




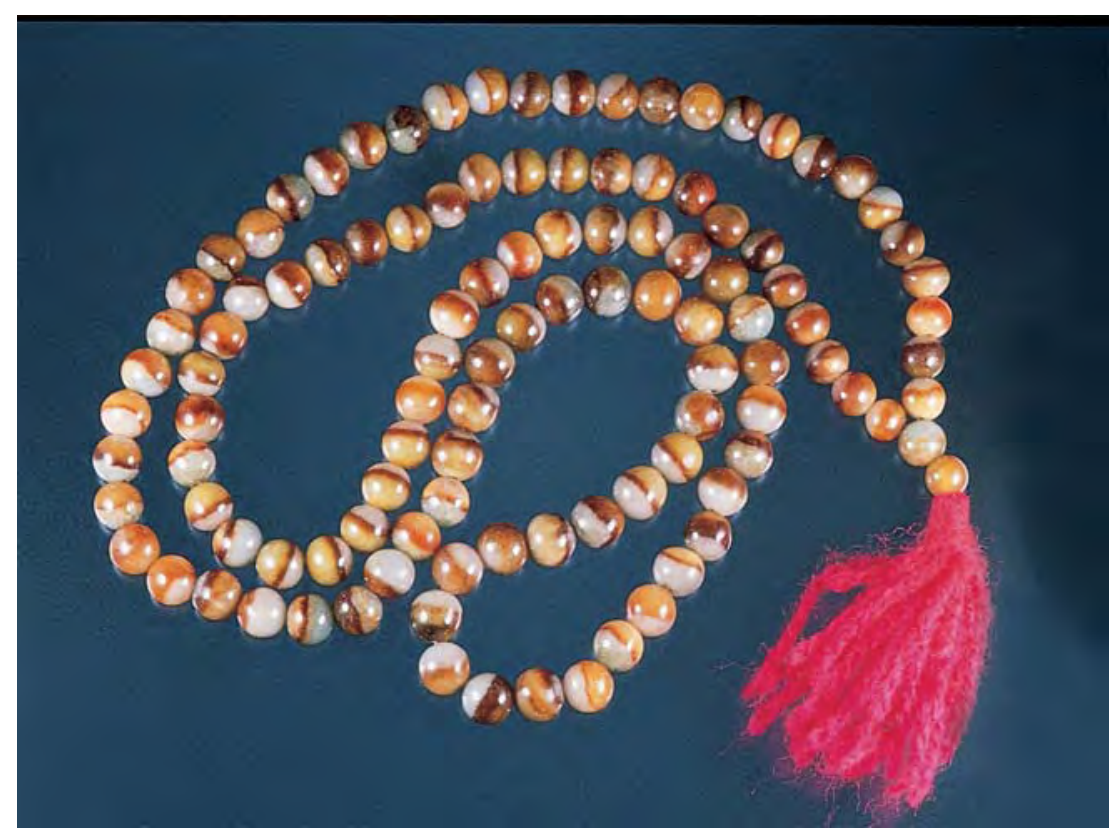

Figure 10. These variegated brown, white, and light green beads (ranging from 8.00 to $10.20 \mathrm{~mm}$ ) proved to be jadeite.

ing. Although many jadeite boulders have a brown outer layer (or "skin"; see, e.g., figure 6 of Winter 1995 Gem News, p. 278), where the color is due to staining by an iron compound, we do not recall ever having seen beads with this agate-like appearance.

Some years ago, we encountered solid-color brown jadeite cabochons that we believed were the result of such natural staining. More recently we have seen jade of a fairly uniform brown color that appeared to be the result of some type of dyeing process. However, the variegated color of these beads indicates natural color. The fact that a number of the beads in the necklace were banded with grayish green jadeite further supports the conclusion that the color was probably natural and that the beads were cut from near the outside layer of a naturally stained jadeite boulder.

Of the beads that we tested, all exhibited a $437 \mathrm{~nm}$ iron line and spot refractive index values within the normal published range for jadeite (about 1.66). For the most part, the beads were inert to both long- and short-wave ultraviolet radiation. The attractive brown-and-white variegated troscopy on a few of the beads confirmed that they were not polymer impregnated.

GRC

\section{Cavity Impregnated \\ With a Colored Filler}

Late last year, the West Coast lab was asked to test a bangle bracelet for polymer impregnation. The translucent green bangle, which measured $63.6 \mathrm{~mm}$ in diameter and $7.93 \mathrm{~mm}$ thick, showed properties typical of jadeite, with blocky grains visible with magnification. Infrared spectra taken on several regions of the bangle demonstrated that it was indeed impregnated with a polymer.

However, bleaching and impregnation was not the only treatment that the bangle had received. A relatively large cavity in the bangle had been filled with a polymer-like substance that fluoresced moderate white to long-wave UV and weak green to short-wave UV. With magnification, this substance was seen to contain numerous tiny green spherules as well as many gas bubbles (figure 11). We suspect that a green coloring agent was added so that the filling material more closely resembled the green jadeite. It certainly made the filled cavity less evident with a cursory examination.

We have previously reported on a Figure 11. The green spherules in
this polymer-filled cavity in a jadeite bangle make the filler much less conspicuous. Note also the many gas bubbles in the polymer. Magnified 25x.

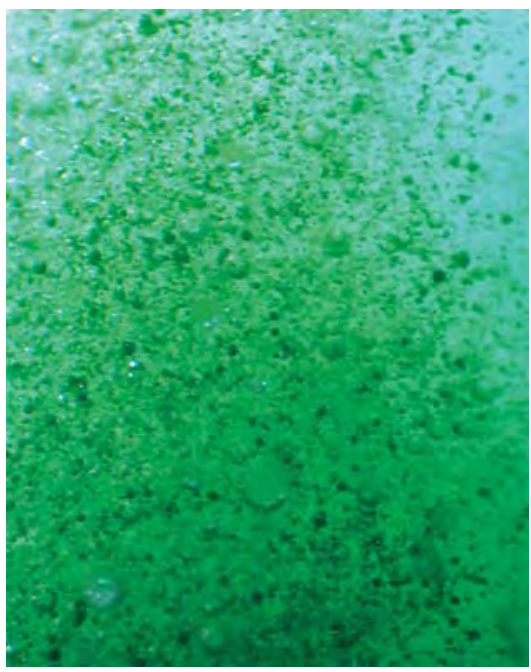
polymer-like substance filling the cavities in a bangle (Winter $1994 \mathrm{Lab}$ Notes, pp. 266-267) and used to repair a jadeite carving (Spring 1996 Lab Notes, pp. 46-48). In addition, we have seen a natural stone bead that could have been mistaken for jade with a polymer-filled cavity but in fact was grossular garnet with a softer chlorite-group mineral (Spring 1996 Lab Notes, pp. 45-47).

$M L J$ and SFM

\section{Dyed Impregnated Bangle without a "Dye Band" Spectrum}

As noted in previous entries, significant treatments used on jadeite jade are dyeing, and bleaching followed by impregnation with a polymer or wax. When such treatments are present, the Gem Trade Laboratory notes them explicitly on Identification 


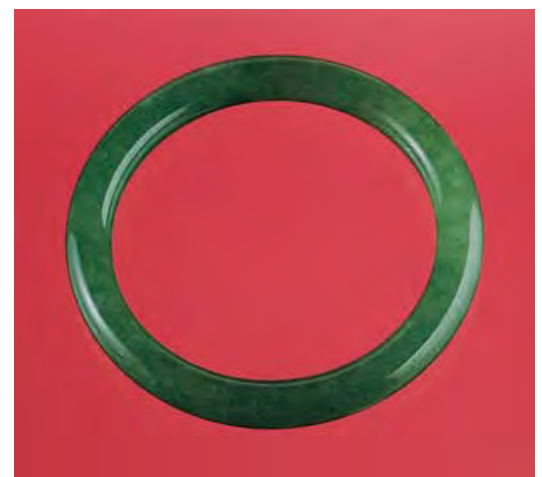

Figure 12. Although testing proved that this jadeite bangle had been dyed and polymer impregnated, examination with a handheld spectroscope did not reveal the broad absorption band centered at $640 \mathrm{~nm}$ that is typical for dye.

reports. In the case of bleaching and impregnation, infrared spectroscopy is usually needed to identify the treatment conclusively. However, most green dye treatments can be detected by two features revealed during standard gemological examination: (1) color concentrations seen (using magnification) along the boundaries between the jadeite grains, and (2) an absorption "dye band" visible between 630 and $660 \mathrm{~nm}$ (as seen, for instance, with a handheld spectroscope).

In spring 1997, a mottled green bangle bracelet measuring $72.6 \mathrm{~mm}$ in diameter by $8.21 \mathrm{~mm}$ thick (figure 12) was sent to the West Coast laboratory for identification. The bangle had an aggregate optic character, with a refractive index of 1.66. It fluoresced a weak greenish yellow to long-wave ultraviolet radiation, but was inert to short-wave UV. With magnification, the material showed an aggregate structure, with color concentrated along grain boundaries (figure 13) and in some patches. These gemological properties were consistent with jadeite jade that had been dyed green. However, no dye band was visible when the sample was checked with a handheld spectroscope! Nor did we see the three bands (at about 630, 660,

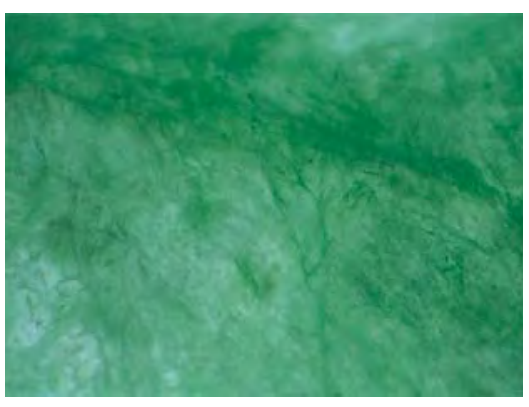

Figure 13. With $20 \times$ magnification, dye concentrations between grains could be clearly discerned in the bangle shown in figure 12.

and $690 \mathrm{~nm}$ ) indicative of chromium in jadeite. Instead, we saw a line at $437 \mathrm{~nm}$ (typical for jadeite), a weak band at $600 \mathrm{~nm}$, and a cutoff edge at $650 \mathrm{~nm}$. This spectrum was very unusual, since in our experience jadeite with the depth of color of this bangle should have had either a chromium spectrum, if natural color, or a broad band centered at about 640 $\mathrm{nm}$, if dyed.

We took an infrared spectrum, as we do for all jadeite samples, and found that the bangle had been polymer impregnated. However, because of the lack of a distinctive optical absorption spectrum, we also checked the chemistry using EDXRF spectroscopy. Again, there was no evidence of chromium. We concluded that the bangle was dyed impregnated jadeite jade.

Figure 14. The $8.64 \times 6.51 \mathrm{~mm}$ doublet in this white metal ring consists of a synthetic spinel crown and a synthetic ruby pavilion.

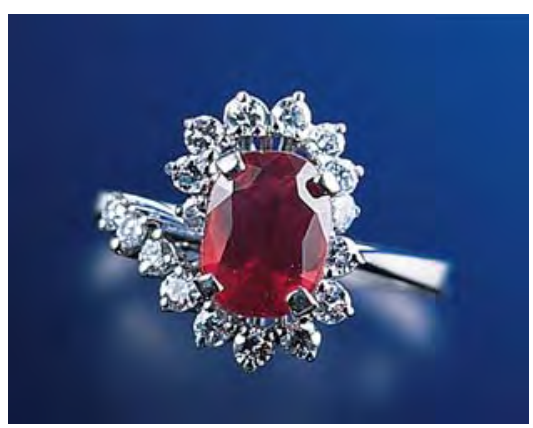

This is the first instance we can recall where a dyed green jadeite piece did not show the typical dye band in the handheld spectroscope. In light of this development, we strongly advise gemologists to subject all green jadeite to careful microscopic examination.

MLJ, SFM, and Dino DeGhionno

\section{RUBY,}

\section{Assembled Imitation}

Last summer, at the same time that the East Coast lab was testing the synthetic green spinel/natural ruby assemblage (represented as spinel) that was reported in the Winter 1996 Lab Notes (p. 281), the West Coast lab was asked to identify the center red stone in the ring shown in figure 14. The white metal ring was stamped " $18 \mathrm{~K}$," and the center stone was surrounded by numerous near-colorless round brilliants. Nevertheless, the red oval mixed cut was easily seen to be a doublet consisting of a near-colorless crown cemented to a red pavilion.

The gemological properties of the crown were: R.I. $=1.728$, singly refractive, medium chalky yellow fluorescence to long-wave UV and strong green fluorescence to short-wave UV. With magnification, we saw small gas bubbles in thread-like arrangements (figure 15). These properties are typical for synthetic spinel, particularly the fluorescence and inclusions. The

Figure 15. Characteristic thready gas bubbles in the synthetic spinel crown and curved striae in the synthetic ruby pavilion are visible in the doublet shown in figure 14 at $40 \times$ magnification.

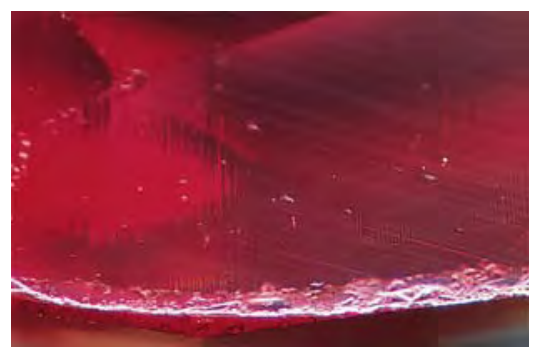


pavilion was doubly refractive, fluoresced moderate red to both long- and short-wave UV radiation, and revealed both curved striae and small gas bubbles with magnification (again, see figure 15). The absorption spectrum, which could only be observed for the doublet as a whole, was typical for ruby. These properties identified the pavilion as synthetic ruby.

A similar doublet was described in the Winter 1984 Lab Notes section (pp. 231-232), but that example was 20 ct. Because of the smaller size of this doublet, and the nature of the mounting, it would more easily be mistaken for a natural ruby. Twelve years after our first encounter with this type of assemblage, we still don't know why such an odd choice of materials was used.

$M L J$ and SFM

\section{SAPPHIRE, With Evidence of Heat Treatment}

A large proportion of the corundum gems, both ruby and sapphire, seen in the GIA Gem Trade Laboratory have shown evidence of heat treatment. The presence of diagnostic inclusions makes identification of this treatment a straightforward process in many cases. Recently, the East Coast laboratory received a 2.14 ct blue sapphire for identification. The stone contained fine examples of inclusions typical of heat treatment (figure 16). For example, a discoid fracture surrounding a mineral inclusion was prominent under the table; and silk (rutile needles), visible throughout the stone, had been partially resorbed and reduced to strings of "pinpoints." The presence of a small melted cavity with a "fire-skinned" surface, as well as the stone's chalky fluorescence to short-wave UV, provided further evidence of heat treatment.

In addition to these typical features, we saw a very unusual raised area at the culet (figure 17). Careful examination of the culet area with magnification (and with both longand short-wave UV radiation) strongly suggested that this raised area was part of the original heat-treated surface of the stone. Such areas are usually removed when a stone is repolished after heat treatment, so that only melted surface areas recessed in cavities may remain. Apparently, the repolishing of this stone after treatment was incomplete.

Additional damage of a quite different appearance was also evident on the pavilion. Rounded spots that had been etched on several pavilion facets (again, see figure 17) had the same appearance as the type of damage sometimes caused by improper jewelry repair. Because corundum is soluble in the heated borax-based fluxes used in jewelry repair, such etched areas can occur on a stone that was not removed from the setting during a repair to the mounting (see, e.g., Summer 1982 Lab Notes, p. 106).

Elizabeth Doyle

\section{SERENDIBITE, \\ A Rare Gemstone}

Last autumn the East Coast laboratory received an identification challenge that turned out to be a truly rare gemstone. The 0.35 ct dark green emerald cut had the following properties: Refractive indices were 1.697 and 1.704. A biaxial optic figure was visible through the pavilion, and the stone showed pleochroic colors of deep blue and pale yellowish green.

Figure 16. A discoid fracture surrounding a mineral inclusion and the partially exsolved rutile needles prove that this $2.14 \mathrm{ct}$ sapphire was heat treated.

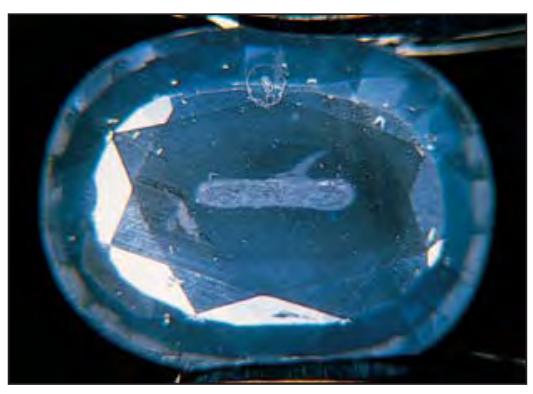

The only features seen with magnification were some transparent to white fingerprint-like inclusions. No fluorescence was observed to either long- or short-wave UV, and a deskmodel spectroscope revealed only a weak line at $470 \mathrm{~nm}$. Because of the stone's small size, we used a noncontact measurement device (Sarin's DiaMension) to measure the volume of the stone and then computed the specific gravity to be about 3.39 .

Although this set of properties ruled out most common gemstones, a careful search of published mineralogical data yielded four minerals that had gemological properties in the observed range: zoisite, dumortierite, serendibite, and sapphirine. The piece was unlikely to be dumortierite, because this material is almost never found in transparent pieces large enough to fashion a 0.35 ct stone. Also, the green zoisite reported to date has different pleochroic colors and absorption spectra (see, e.g., N. R. Barot and E. W. Boehm, "Gem-quality Green Zoisite," Gems « Gemology, Spring 1992, pp. 4-15). We next turned to advanced testing techniques to determine whether it was sapphirine, serendibite, or even a possibility that we had not yet considered.

Although the structures of these two minerals differ enough that one could expect their infrared spectra to

Figure 17. The melted surface at the culet of the stone in figure 16 is higher than the surface of the facets, suggesting that the culet was not repolished after treatment. Note also the rounded features that have been etched on some pavilion facets. Magnified 13×.

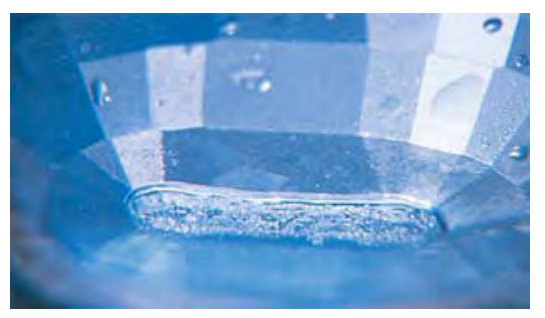


be distinct, both sapphirine and serendibite are so rare that we did not have reference spectra or even reference stones to which we could compare the spectrum we took. An EDXRF chemical analysis showed silicon, aluminum, magnesium, calcium, iron, and minor amounts of titanium, which is consistent with the chemical formula for serendibite. Xray diffraction analysis proved conclusively that the gem was serendibite.

Although first described in 1902, serendibite is so rarely seen as a gemstone that it is not found in most gem reference books. We could find no previous mention of it in Gems $\leftrightarrow$ Gemology, but some of GIA's most senior gemologists recall seeing one specimen in the late 1960s or early 1970s.

The name of this mineral comes from Serendib, the old Arabic word for Sri Lanka. This same place name, and a fairy tale about three lucky princes from that island nation, led Horace Walpole to coin the word serendipity in the mid-18th century. It was certainly serendipitous that this stone came to our attention.

$I R$ and $M L J$

\section{ZIRCON, With "Play-of-Color"}

Over the years, we have examined and reported on zircons that displayed a variety of phenomena. These have included aventurescence, chatoyancy, change-of-color, and iridescence. It is not often that we encounter a gem material with a phenomenon that has not been previously reported. It was therefore with great interest that we examined a dark brown zircon cabochon that displayed what appeared to be play-of-color. The gemological properties of this cabochon were typi-

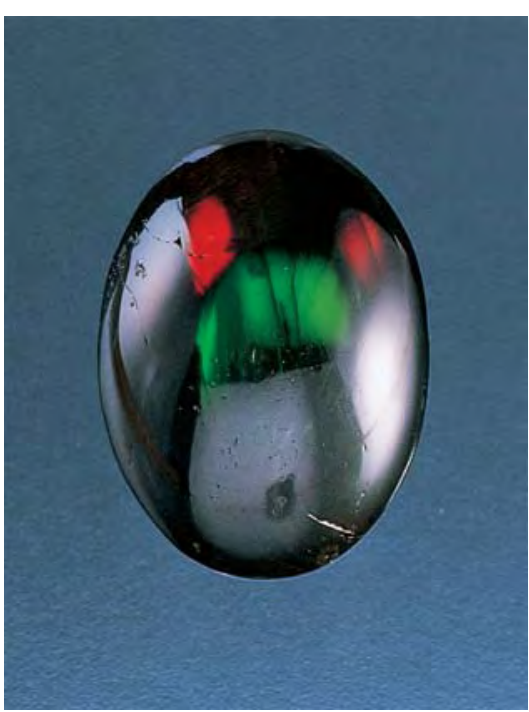

Figure 18. This $12.05 \mathrm{ct}$ brown zircon shows a play-of-color similar to that seen in opal.

cal for zircon: The R.I. was over the limits of the refractometer, the S.G. was 4.15 , it was inert to long-wave UV but fluoresced a weak slightly chalky yellow to short-wave UV, and the absorption spectra showed several distinct lines dominated by a pair at $635 \mathrm{~nm}$

The first thing we noticed on visual inspection were flashes of green and red emanating from several areas of the cabochon (figure 18). We would normally expect such colors in zircon to be iridescence coming from fractures, or perhaps from a finely laminated structure (see Gems e) Gemology, Spring 1990, p. 108). However, on closer inspection we saw no iridescence in the fractures, and we determined that these flashes of color were coming from threedimensional regions within the body of the stone. These regions were sharply defined and showed no evidence of a laminated structure (figure

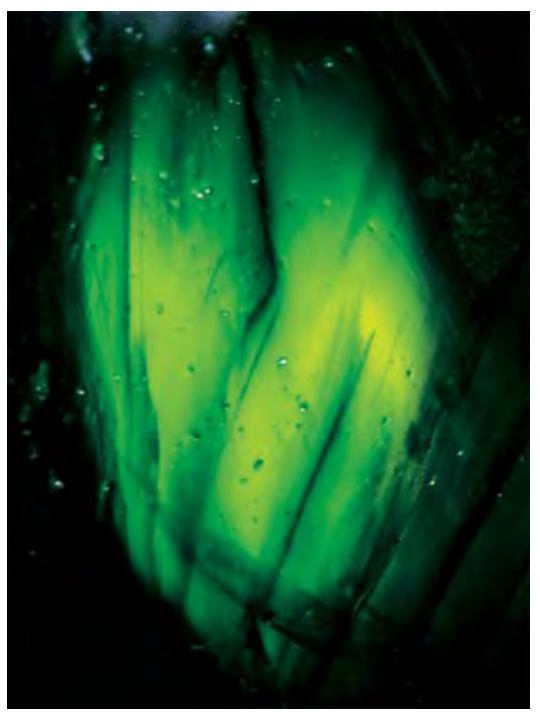

Figure 19. The play-of-color seen in the zircon shown in figure 18 was confined to sharply defined three-dimensional zones, proving that the phenomenon was not due to iridescence from fractures or diffraction from a laminated structure. Magnified 30x.

19). The resemblance of this phenomenon to the play-of-color found in opal was striking and unlike anything we had seen before in zircon. Unfortunately, we could not determine the cause of this phenomenon, because we were not able to perform the necessary structural analysis of the stone in the time that we had available.

SFM

\section{PHOTO CREDITS}

Shane McClure provided figures 1, 11, 13, 15, 18, and 19. The photos in figures 2, 3, and 6 were taken by V. J. Cracco. Nick DelRe supplied the pictures used in figures 4-5, 7-8, 10, and 16-17. Figures 9, 12, and 14 were taken by Maha DeMaggio. 